\title{
La lexicographie associative : vers un nouveau dictionnaire français des associations évoquées par les mots
}

\author{
Michèle Debrenne ${ }^{1}$ \\ ${ }^{1}$ Université d'Etat de Novossibirsk, 630090 Novossibirsk, Russie \\ micheledebrenne@gmail.com
}

\begin{abstract}
Résumé. L'article présente une nouvelle étape du projet de création du Dictionnaire associatif du français, dans lequel sont notées les associations évoquées par les mots telles qu'elles sont collectées grâce à des questionnaires de fixation de la première réponse à un stimulus. Nous nous proposons de tenir compte de l'expérience acquise au cours des dix dernières années et des avancées des humanités numériques pour automatiser au maximum la collecte et surtout le traitement des grands massifs de données, de les comparer avec les données précédentes et de visualiser les résultats obtenus.
\end{abstract}

\begin{abstract}
Associative lexicography : toward a new dictionary of word associations of French The paper presents a new step of creation of the French Dictionary of Word Associations, in which associations to a stimulus are collected. Our team is working in this field from 2007 and aims to use their obtained skills, correct some errors and collect new language facts. The use of modern digital technologies for humanities allows to collect and treat big amount of data, to compare them with previous results and visualize them.
\end{abstract}

\section{Etat des lieux}

\subsection{Dictionnaire des Associations du Français (DAF)}

L'étude des normes associatives de la langue française a commencé en 2006, avec la première version intitulée "Dictionnaire des associations verbales du français », projet interuniversitaire franco-russe. La conception du dictionnaire était basée sur celle des projets russes analogues, où la lexicographie associative est bien développée et documentée [voir 1-14]. Ainsi, les participants à l'expérience étaient tous des étudiants. Mais sous d'autres aspects ce dictionnaire différait de ses analogues :

- au lieu d'un questionnaire papier rempli en présence du chercheur, le choix avait été fait en faveur d'une expérimentation en ligne sur un site dédié. Chaque participant à l'expérience devait, en un temps limité, écrire ce qui lui passait par la tête (mot, expression) en réaction à 100 stimuli (mots pleins de la langue française). A la différence du test papier traditionnel ils ne voyaient qu'un stimulus à la fois et ne pouvaient pas revenir en arrière. 
La liste de stimuli était générée aléatoirement pour chaque participant d'après une liste totale de 1100 mots, choisis parmi les plus fréquents de la langue française.

- comme il avait été prévu de comparer les associations fournies par les participants en France métropolitaine et en Afrique francophone, une centaine de stimuli avaient été rajoutés, qui, semblait-il, devaient être ressentis de manière différente dans ces deux zones linguistiques, par ex. case, boy, chicotte, boubou. Ce dernier stimulus, effectivement, a fait l'effet d'un «bruit» privé de sens pour la plupart des francophones de France métropolitaine, qui ont réagi par d'autres onomatopées ou par le nom de personnages d' « animés » japonais. Malheureusement jusqu'à l'heure actuelle l'expérience en Afrique n'a pas pu être menée à bien.

Même si elle s'est conclue par la publication du dictionnaire direct (du stimulus à la réaction) et inverse (de la réaction au stimulus) et d'un site internet, activement utilisés en Russie dans des travaux à visée comparatiste, il reste évident que cette première expérience a des défauts, qui devront être évités dans le cadre du projet actuel.

\subsection{Dictionnaire des normes associatives de la Francophonie (DINAF)}

La seconde étape de l'étude s'est concrétisée par celle des normes associatives de la francophonie. Elle a été provoquée par une discussion animée autour du « néohumboltianisme » russe et la critique de la lexicographie associative [15-20] à la suite de laquelle il a été décidé de vérifier si les normes associatives sont conditionnées par la langue ou par l'environnement sociolinguistique. Une expérience de moindre envergure que la précédente (portant sur une liste de 100 stimuli, les mêmes pour tous) a été menée en France, Belgique, Suisse romande et Canada francophone, à l'aide d'un questionnaire Google. A la différence de l'expérience présentée en 1.1, tout le monde, et pas seulement des étudiants, pouvait participer à la seconde, sans limite d'âge ou de profession. Malgré une méthode de diffusion semblable, les résultats ont été inégaux dans les quatre zones géographiques choisies, et il n'a pas été possible en deux ans de réunir pour la Suisse romande les 500 réponses souhaitées [21]. Les données récoltées ont été cependant publiées sur internet, où on peut visualiser et comparer les champs associatifs des différents locuteurs du français. Les études menées sur ce corpus n'ont pour l'instant pas mis en évidence de différences significatives [22].

\subsection{Autres projets de lexicographie associative pour le français}

Les associations évoquées par les mots ont été également utilisées (en complément d'autres données fournies par des questionnaires) pour la création d'un autre projet de lexicographie associative et naïve, le «Dictionnaire usuel des bionymes ». Les articles du dictionnaire comprennent le mot-vedette puis, présentés par ordre de fréquence décroissante, les réactions à un test d'association, les définitions données par les usagers de la langue (qualifiées ici de naïves), les phrases associées et les comparaisons. Le projet pilote pour le français porte sur les substantifs suivants : âne, chou, corbeau, coq, lièvre, loup, mouche, rose, souris, veau.

Ces travaux se placent au cœur de la tendance manifestée des 20 dernières années en Russie et que l'on désigne généralement par " principe anthropocentrique », se consacrant plus au locuteur (ou à son interlocuteur) qu'au système de la langue. Outre la théorie de la linguistique naïve, ce principe a donné naissance, entre autres, à des études consacrées aux concepts en sémantique, ou à celles qui s'organisent autour de la personnalité linguistique ou du portrait discursif [23]. Dans ce dictionnaire le principe d'anthropocentrisme n'est pas simplement déclaré, il est véritablement au centre de l'étude, puisque c'est bien la sémantique de l'homo loquens qui est systématisée ici, la signification psychologique réelle, l'expérience de communication au sein de la communauté de langue. Cet ouvrage 
montre réellement le fonctionnement de ce que les chercheurs russes nomment «conscience linguistique » des locuteurs du russe actuel, du moins de ceux qui ont participé à l'expérience. Les articles de ce genre de dictionnaire sont très riches en enseignements. Ils sont plus informatifs qu'un simple dictionnaire d'associations, dont les articles laconiques sont parfois énigmatiques.

\section{Pourquoi un nouveau dictionnaire des associations évoquées par les mots pour le français (NDAF) ?}

\subsection{Critique des projets précédents}

Nous considérons qu'il est temps de proposer un nouveau dictionnaire des associations évoqués par les mots pour le français. En effet, grâce à sa participation dans les projets évoquées plus haut, l'équipe a acquis un savoir-faire certain dans le domaine de la collection et du traitement des données issues d'expérimentations psycholinguistiques. Les défauts des dictionnaires précédents sont apparus, auxquels il convient de pallier. Les progrès des humanités digitales, les possibilités de prendre en compte d'immenses masses de données doivent être utilisées. Par ailleurs, dix ans se sont passés depuis la première expérimentation, une nouvelle permettra de comparer, dans une perspective diachronique, ce qui est stable et ce qui est éphémère dans les champs d'associations.

Les défauts du dictionnaire des associations du français à éviter dans la nouvelle version sont les suivants :

La solution choisie pour éviter les homonymies et la polysémie de certains stimuli n'a pas donné les résultats escomptés [24]. En effet, dans certains cas, l'article avait été ajouté au stimulus, par ex. bien / le bien. Cependant, étant donné que chaque participant à l'expérience était confronté à une liste originale, la probabilité d'avoir les deux formes dans la même expérience était très faible. Ainsi, si les personnes confrontées au stimulus le bien réagissaient au substantif, celles qui rencontraient bien n'avaient aucun moyen de savoir s'il s'agissait de l'adverbe ou du substantif, ce qui s'est reflété dans le champ associatif. La solution choisie n'avait donc pas permis de limiter les réponses à l'adverbe. De plus, elle n'avait pas été appliquée systématiquement, puisque de nombreux stimuli n'avaient pas été discriminés : par ex. la liste comportait des stimuli tels fort ou neuf qui pouvaient être interprétés non pas comme des adjectifs, mais également comme un fort ou le chiffre neuf. Il est évidemment impossible de prévoir toutes les ambigüités du lexique français et il semble préférable dans cette version ultérieure du dictionnaire de traiter tous les stimuli de la même manière, laissant aux participants le choix des significations ou des lemmes.

Le défaut principal du premier projet est, à notre avis, que pour l'établissement de la liste de stimuli de départ il n'a pas été possible de bénéficier de travaux contemporains sur la fréquence des lexèmes en français. Les dictionnaires existants étaient anciens et basés sur des corpus de taille limitée, et la liste de stimuli a été basée sur une liste de fréquence proposée par le Ministère de l'Education nationale pour l'enseignement du français à l'école. Depuis, un dictionnaire de fréquence basé sur l'étude de 23 millions d'unités lexicales est paru [25] et il est indispensable de le prendre en considération dans les travaux ultérieurs. En effet, il semble important d'utiliser dorénavant une liste de stimuli établie sur une base scientifique solide, même, comme on pouvait l'espérer, les listes de fréquence ne sont pas entièrement différentes. Ainsi, 95 des stimuli utilisés pour l'élaboration du DINAF se retrouvent dans les 100 premiers du dictionnaire Lonsdale et LeBras et si on compare la liste utilisée dans le DAF et les 1000 premiers mots pleins de Lonsdale et LeBras, on constate 611 coïncidences (avec un rang différent). Ces coïncidences permettront d'effectuer des comparaisons entre les deux dictionnaires. 
Enfin la version papier du DAF souffrait de défauts techniques dus à une prise en compte défectueuse des lettres accentuées, ce qui a faussé la présentation par ordre alphabétique, défaut heureusement corrigé dans la version électronique du dictionnaire.

\subsection{Projets voisins}

Outre les dictionnaires édités en Russie, dans l'établissement du NDAF il convient de prendre en compte un certain nombre de projets similaires développés pour cette langue.

Il s'agit en premier lieu du projet porté par l'Université de Louvain, A small world of words porté par Gert Storms et Simon de Deyden [26]. C'est un dictionnaire "classique » d'associations (direct et inverse) dont le but déclaré est de construire un dictionnaire mental. Les principes de base de l'expérience sont les suivants : 18 mots (stimuli) proposés par participant, trois réactions exigées (au lieu d'une seul dans l'expérience du DAF). Cette différence n'est pas sans conséquences - en effet, on a constaté que seule la première réaction est relativement spontanée ; les réactions suivantes sont provoquées non seulement par le stimulus, mais également par la réaction donnée précédemment [27]. Ainsi une expérience en chaine comme celle utilisée dans le projet de Louvain montre plus le processus mental du locuteur que la structure en réseau du lexique. Les principales langues de l'expérience sont l'allemand et le néerlandais, et si le site propose un certain nombre d'autres langues (anglais, espagnol et espagnol rioplatense, italien, portugais, chinois et cantonais, japonais, russe, vietnamien), le nombre de participants à l'expérimentation est très différent selon les langues. Ainsi, seules 921 personnes ont pris part à l'expérience en français, ce qui a permis de récolter 33024 réactions.

Le second projet est celui de Mathieu Lafourcade, Jeux de mots [28]. Le but de la collecte de données est de construire un réseau lexical dans lequel les relations entre le stimulus et la réaction sont étiquetées (par exemple voler : agent/ oiseau) qui sont ensuite utilisées dans des systèmes de TAL. L'expérience a été lancée il y a dix ans et a récolté un nombre très impressionnant de paires stimulus-réaction (90 552430 au 8 juillet 2017), nombre en constante augmentation, car le site fonctionne activement, grâce à sa présentation sous forme de jeu ; le participant à l'expérience choisit lui-même le nombre de mots auquel il veut donner des réactions (il peut «sortir du jeu » à tout moment), il ne remplit aucun questionnaire permettant une étude statistique ultérieure (tel sexe, âge, localité, langue maternelle). En outre, la grande différence entre ce jeu et les autres expérimentations connues d'associations évoquées par les mots est que le sujet d'expérience doit en un temps limité donner le plus de réactions possibles au stimulus. Des règles (compliquées) permettent d'obtenir du temps supplémentaire. Le but du jeu est à la fois de donner des réactions originales, et qu'elles coïncident avec celles d'autres participants. Tout cela permet de conclure que cette expérience ne peut pas être comparée avec les dictionnaires d'associations «classiques» car les consignes données aux participants faussent la spontanéité exigée dans les autres études. Toutefois le fait que les données obtenues dans le cadre de ce projet soient en accès libre permettra d'effectuer des comparaisons intéressantes.

En outre, pour faciliter le traitement ultérieur des données, le site Jeudemots permet un traitement manuel par le sujet d'expérience : en effet, on lui propose de préciser le sens de la réaction qu'il a donnée, en outre le système rejette toute orthographe non conforme. Ceci est proche de certains des principes qu'il est proposé d'implémenter dans notre nouveau dictionnaire.

\subsection{Principes de base du NDAF}

\subsubsection{La liste de départ}


Une liste de 1000 mots pleins (uniquement composée de substantifs, verbes et adjectifs) sera proposée, tirée du dictionnaire de fréquence de Lonsdale et LeBras. Dans cette liste le dernier mot, université, a un rang de 1192. Au fur et à mesure que l'expérience se déroulera, les stimuli qui auront atteint un nombre suffisant de réactions validées, fixé à 500 , seront remplacés par de nouveaux stimuli, tirés du «noyau de la conscience linguistique ", selon la terminologie russe c'est-à-dire les réactions provoquées par le plus grand nombre de stimuli - "grands connecteurs » dans la terminologie des réseaux petits mondes [29]. Cette méthodologie est inspirée de celle utilisée par Karaulov et son équipe pour la création du « Thésaurus associatif du russe » [30].

L'homonymie et la polysémie des stimuli ne sera pas désambigüisée, ainsi page peut référer aussi bien au jeune homme qu'à la feuille de papier, pièce pourra évoquer le théâtre, la monnaie, les parties d'un appartement et beaucoup d'autres choses encore. De même, le fait que la liste soit limitée aux substantifs, verbes, adjectifs ne sera pas communiqué aux participants à l'expérience, et rien ne les empêchera d'appréhender des stimuli tels ton, or, bien comme d'autres parties du discours (déterminant, conjonction ou adverbe).

\subsubsection{La collecte des données}

Comme précédemment, la collecte de la matière se fera sur internet, en utilisant ce qui est aujourd'hui communément appelé la «production participative» (crowdsourcing). Une approche ludique étant plus attractive qu'un projet à visée scientifique annoncée, quelques modifications seront apportées au site d'origine, à savoir notamment :

Une liste de stimuli limitée à 25 par personne, sachant qu'il est difficile de retenir un visiteur d'un site plus de 5 minutes.

La possibilité de visualiser immédiatement comment les réponses de cette personne correspondent à celles des autres participants (pour l'ensemble des stimuli ou pour un stimulus donné), car ce genre de question est souvent posé par les participants à l'expérience: un test psycholinguistique, quelque ludique qu'il soit, ouvre une porte à l'inconscient linguistique de la personne, et toute personne qui en a passé ou fait passer est confrontée à des réactions du type «j'ai bon?» ou, au contraire «mes réponses sont totalement originales, vous ne pourrez rien en faire de bon ». Une visualisation permettra donc de satisfaire à la curiosité du sujet d'expérience.

De même, les participants à l'expérience auront la possibilité de visualiser le champ linguistique d'un stimulus, sous la forme d'un nuage de données ou d'un réseau lexical. Au début, tant que la nouvelle base de données ne sera pas complétée, la visualisation sera celle des résultats des expériences précédentes.

L'expérience sera précédée d'un minimum de questions sur le sujet : âge, sexe, pays de résidence, langue maternelle. Une question ouverte sur les autres langues connues du sujet d'expérience sera ajoutée par rapport aux expérimentations précédentes. En effet, l'influence du bilinguisme-plurilinguisme sur les réactions d'associations évoquées par les mots est un sujet d'actualité qui jusqu'à présent n'a pas pu être correctement traité par manque de données fiables. Il convient par ailleurs de placer ces questions en début et non en fin d'expérience, pour éviter d'être obligés de mettre au rebut des formulaires incomplets, ce qui avait été le cas pour le DINAF.

L'attractivité du site sera augmentée par une série de brèves publications "grand public » relayées sur les réseaux sociaux (voir infra §2.4). On pourra y trouver l'analyse du champ associatif (ensemble des réactions provoquées par un mot) et du champ des stimuli (ensemble des stimuli ayant provoqué cette réaction), pour divers mots, la comparaison de ces données entre différentes régions de la Francophonie (voir fig.1), ou avec d'autres langues, la comparaison de la liste des « grands connecteurs » et/ou des réactions les plus fréquentes en Francophonie, ainsi que des articles sur des observations amusantes sur, par exemple, les jeux de mots et calembours (trou $\rightarrow$ badour ou pied $\rightarrow$ d'estal), les fautes de 
frappe et d'orthographe, les noms propres et références culturelles. Ces articles inciteront d'autres usagers à visiter le site et participer à l'expérience, comme ce qui se fait, par exemple, pour le site Français de nos régions [31].

\author{
Stimul: mer \\ Database: (- DINAFN DINAF \\ Database: $\square$ display "joint" \\ Refresh
}

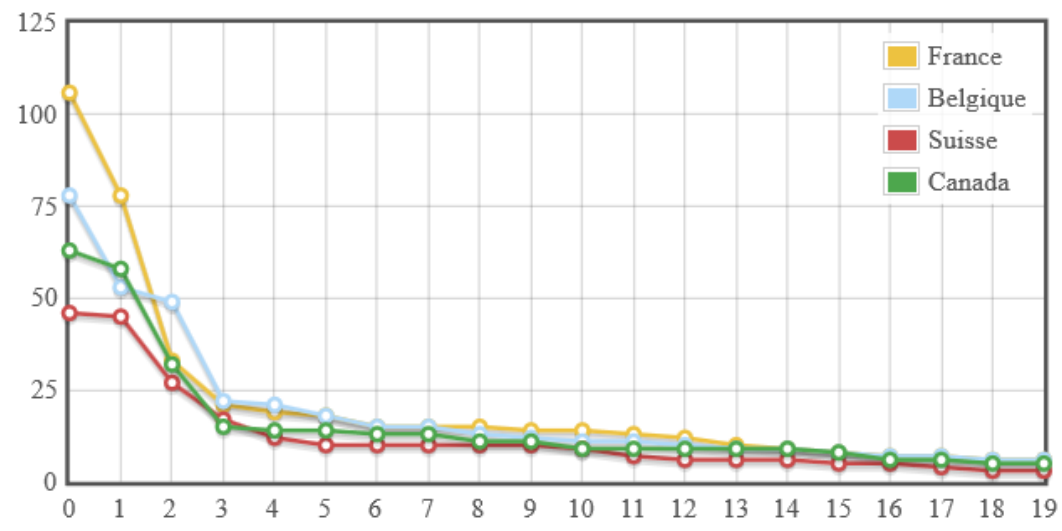

Fig. 1 Comparaison des réactions données en France, Belgique, Suisse et Canada pour le stimulus mer.

\title{
2.3.2 Le traitement des données
}

Afin d'être en mesure de proposer une visualisation et une exploitation immédiate des données, celles-ci doivent être retraitées automatiquement dès la saisie. La problématique est la suivante : d'une part il convient de conserver les données brutes telles qu'elles ont été entrées par les participants à l'expérience, de manière à préserver l'objectivité scientifique et à être en mesure de désambigüiser des réponses problématiques. Cette matière brute est également utile pour l'étude des erreurs orthographiques, des fautes de frappe (par ailleurs de plus en plus limitées par les correcteurs automatiques inclus dans les gadgets électroniques) des sujets de l'expérience, ainsi que l'analyse de l'aspect syntagmatique et paradigmatique de leurs stratégies de réaction [32]. La pratique russe de création de dictionnaires d'associations évoquées par les mots est de corriger si possible l'orthographe, mais de conserver les formes d'un lemme et de les comptabiliser comme des réactions différentes (par ex. blanc, blancs, blanche et blanches). Cela est également valable pour les figements, qui sont relativement nombreux parmi les réactions, cf. pour le stimulus reprendre : voler ; donner c'est donner, reprendre c'est voler; reprendre c'est voler ; ...c'est voler; c'est volé; c'est voler; pas bien, donner c'est donner; volé. (DINAF joint, sur 1689 réponses). Dans ce type de travaux les regroupements des réactions sont effectuées par la suite, par les chercheurs qui analysent le champ des associations, suivant l'une ou l'autre des méthodes connues.

Dans notre cas, pour obtenir des statistiques exploitables, surtout au niveau du dictionnaire inverse, il convient de regrouper ces réactions au maximum. C'est pourquoi 
nous proposons les stratégies d'unifications suivantes (sachant que les données brutes sont également conservées et exploitables par les chercheurs) :

- tout en interdisant la saisie intuitive, qui fausserait la spontanéité de la réaction au cours de l'expérience, un choix de formes sera proposé au sujet d'expérience a posteriori, notamment pour désambigüiser les formes non accentuées (par ex. pour une réaction orthographiée eleve il sera proposé de cliquer sur élève ou élevé);

- les articles, pronoms personnels ou prépositions sont automatiquement éliminés, ex. la main, une main sont ramenés à main (stimulus donner) ;

- les réponses monolexicales ainsi obtenues sont systématiquement ramenées à leur forme lemmatisée (grande ramené à grand, portes à porte) ;

- les figements, citations et autres expressions proverbiales sont ramenées à la forme statistiquement la plus fréquente. Par exemple, pour le stimulus être, conserver la réaction sous la forme 'ou ne pas être' notamment dans les cas où on avait obtenu des réponses 'ne pas être', 'être ou ne pas être', 'être ou ne pas être voilà la question' etc., moins fréquentes. Pour un traitement automatique de ces situations il convient d'établir une liste de règles basées sur les données obtenues au cours des expériences précédentes.

Notons qu'il convient également d'appliquer systématiquement la réforme orthographique de 1981, de manière à éviter que des réactions telles paraître et paraitre soient prises en compte comme différentes.

Cette politique a été appliquée sur le DINAF qui existe actuellement sous deux formes, l'une après la correction orthographique (les données brutes étant conservées également) et l'autre, dite 'normalisée', après le traitement exposé ci-dessus. Une politique d'unification des données analogues est pratiquée par l'équipe de psycholinguistique de Voronej [33] dans le but d'obtenir des champs d'association dits « intégraux ».

\subsection{Exemple d'article}

Les articles «grand public » publiés sur le site du projet sont basés sur des études entreprises dans le cadre de publications scientifiques, de mémoires de fin d'année d'étudiants, produits pour la plupart du temps en russe. Ils seront illustrés de schémas et de visualisations proposées par le site du projet du «Nouveau dictionnaire français des associations évoquées par les mots ». Ci-dessous nous en proposons un exemple, celui de fête, basé sur les réponses obtenues dans le DAF complétées par celle du DINAF inverse (puisque le stimulus " fête » n'apparaissait pas dans la liste de départ de cette deuxième expérience). Etant donné qu'il s'agit d'un article pour le grand public, un certain niveau d'interactivité est prévu avec des questions adressées au lecteur (par exemple la question Et que penser de ces réactions? qui sur le site sera suivi d'un champ de réponse: votre réponse : ).

\section{Fête}

533 personnes ont donné une réponse à ce stimulus, trois ont refusé, une a répondu rien, une autre non, (ce qui peut être vu aussi comme des refus de réponse). On a obtenu 135 mots différents, dont 80 hapax (mots uniques), ce qui peut être représenté par le nuage suivant (fig.2): 


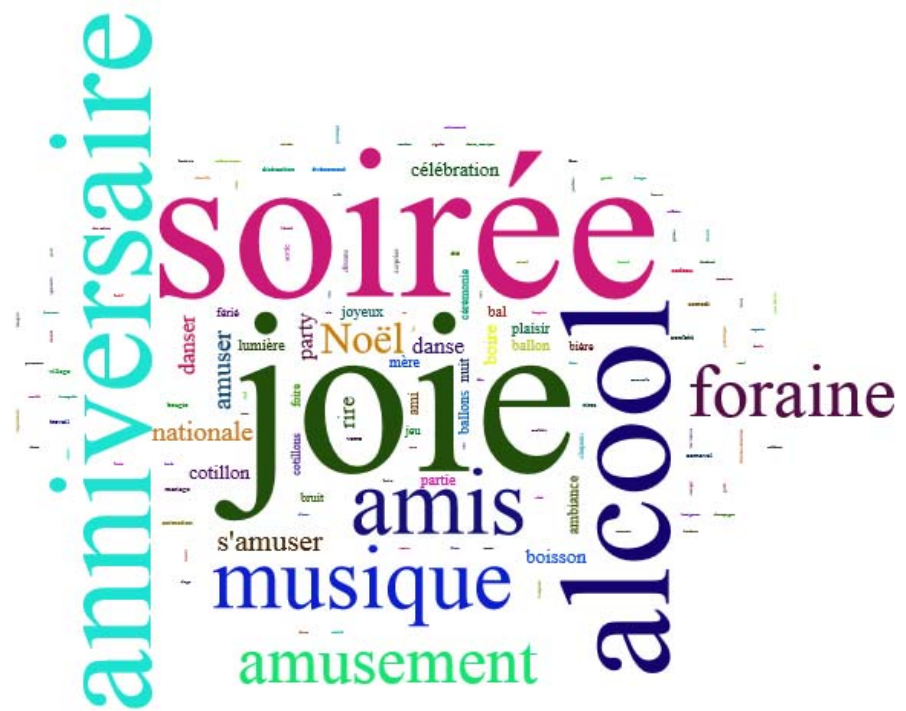

Fig. 2 Nuage de tags visualisant les réactions les plus fréquentes données au stimulus fête dans le DAF.

Les 20 réactions les plus fréquentes sont les suivantes (fig.3):

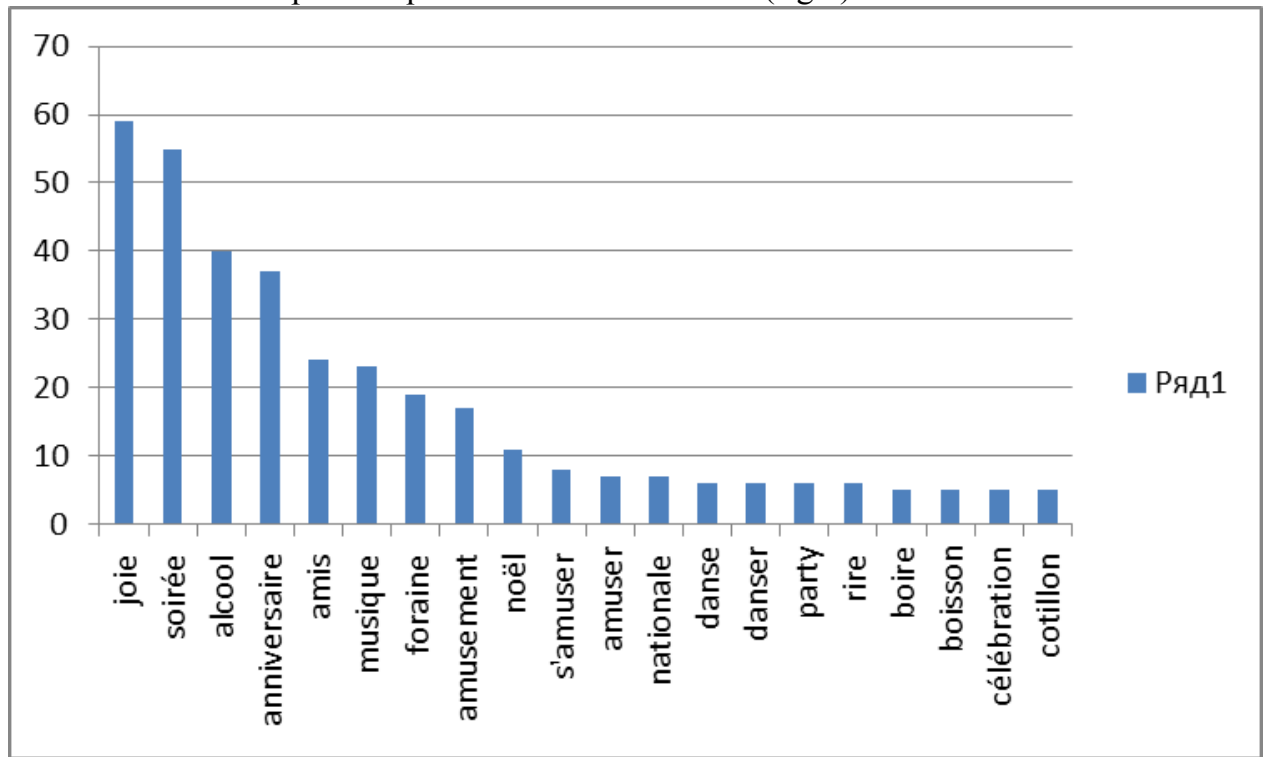

Fig. 2 . Les 20 réaction s les plus fréquentes données au stimulus fête dans le DAF.

L'ensemble des réactions permet de mettre en évidence les regroupements suivants :

Occasions de faire la fête: anniversaire, noël, nationale, bal, des mères, nuit, mariage, samedi, évènement, bar mitzva, jour de l'an, été, printemps, travail, école, village, mensuelle, minuit, occasion, une personne a même considéré qu'un enterrement était l'occasion de faire la fête.

Ce qu'on fait pendant une fête : musique (+trompette), amusement ( + s'amuser), danse (+danser), rire, rigoler, cotillon, chapeau, bal, ballon, lumière, bruit, jeu (jouer), bougie, cadeau, confetti, club (+boite, disco), gâteau, lampion, lumignon, manger, spectacle, youhou. On notera la présence notable des mots qui désignent la boisson: alcool, boire, boisson, boisson alcoolisée, bière, bourré, champagne, vin - en tout 58 
réactions sur 533, auxquelles on peut rajouter Sam - le capitaine de soirée, conducteur désigné qui ne boit pas et ramènera les autres.

Avec qui on fait la fête : ami, copain, amitié, personne, étudiant.

Les sentiments provoqués par la fête: ambiance, joyeux, plaisir, animation, détente, distraction, bonheur, célébrer, cool, couleur, éloge, enthousiasme, fantaisie, festif, gaieté, heureux, liberté, loisirs pittoresque, rare, réjouissance, relâche. Parmi toutes ces réactions positives, on rencontre un ennui, proposé par une personne qui, probablement, n'aime pas faire la fête.

Lieux où on fait la fête : hangar, salle, salon.

Un certain nombre de personnes interrogées ont réagi au mot plus qu'au concept et donné des synonymes du mot "fête»: soirée, amusement, party, cérémonie, foire, carnaval, sortie, surprise, festin, fiesta, réjouissance, soir, teuf, show, rave.

Parmi ces synonymes on trouve un mot régional, chouille, employé en Lorraine.

Une personne a évoqué un verbe, faire (faire la fête), et une autre répondu par assonance tête (fête-tête), et une a continué le figement (fête-)Dieu.

Un certain nombre de réactions n'ont pas trouvé d'explication, les lecteurs de cet article auraient-ils une idée?

fête $\rightarrow$ à bras : Nous supposons qu'il s'agit d'un (mauvais) calembour, basé sur fête $\rightarrow$ fier, qui donne ensuite « fier-à-bras»

fête $\rightarrow$ basses : à rapporter à « musique »?

fête $\rightarrow$ oublié : la personne interviewée aurait-elle oublié de fêter la fête de quelqu'un ?

fête $\rightarrow$ rose on offre des roses à quelqu'un pour sa fête ?

Et que penser de ces réactions ? fête $\rightarrow$ heures, fête $\rightarrow$ monde, fête $\rightarrow$ souvenir?

Le dictionnaire inverse, qui montre quels mots ont fait penser à la fête, confirme ces regroupements sémantiques. On peut affirmer que cette réaction est relativement populaire, puisqu'elle a été provoquée par 78 stimuli différents, avec une fréquence totale de 673 occurrences, ce qui lui confère un bon rang parmi les grands connecteurs - les mots du lexique reliés au plus grand nombre d'autres et qui lui confèrent une structure de type «petit monde».

Ainsi, les mots suivants, partageant le sème commun d'évènement, ont provoqué la réaction "fête": soirée, évènement, occasion, sortir, spectacle, mariage, an, année, national, marier, tradition, traditionnel, coutume, saint (+foi) retour, air avec un score total de 470 paires «stimulus-réaction », c'est-à-dire plus de la moitié des 673 recensées; de même que des mots qui signifient des moments de la journée ou de l'année propices à faire la fête : soir, nuit tard, moment, veille (33); et des lieux où elle peut prendre place : village, salle, place, université (+ étude), cabaret, jardin, théâtre, rue, nord (38).

La boisson est associée à quelque chose de festif, donc bière, boire, boisson, vin (12) ont évoqué la fête chez certains participants à l'expérience. La musique et la danse, elles aussi, semblent souvent se manifester à l'occasion d'une fête et il est donc naturel que les mots suivant aient attiré cette réaction : danser, tambour, chanter, musique, sonner, tamtam, piste, tourner (30); dans certains cas une musique trop forte fait penser qu'une fête se déroule dans les environs : bruit, troubler, agiter (4)

Les émotions positives font penser à une fête: amuser, émotion, énergie, gloire, plaisir, folie, joie, amour éclater? (24)

On fait la fête avec ses amis, et surtout quand on est jeune, donc les mots suivants évoquent la fête : ami, jeune, jeunesse, foule, social (19); et un certain nombre d'attributs peuvent s'y associer : masque, lumière, robe, argent (10).

Quant aux paires "stimulus-réaction » conditionnées par la langue, on peut noter le synonyme partie, l'anonyme deuil et l'expression figée "lendemain de fête » qui s'est rappelée au bon souvenir de 7 personnes. 
Un certain nombre de verbes d'action demandent «fête" comme complément : préparer; réunir, recevoir, retrouver, surprendre, rencontrer, partager (19). Curieusement on ne voit pas apparaitre dans cette liste le «bouche-trou» universel faire (« faire la fête »).

Seul le stimulus plein ne trouve pas d'explication pour l'instant.

Si l'on compare cette liste de mots évoquant la fête dans l'expérience de 2007 et celle obtenue en 2015 dans une zone plus vaste (comprenant en plus de la France hexagonale, la Belgique, la Suisse romande et le Canada francophone), on en retrouve certains, auxquels se rajoutent faire (4 fois), venir, arriver, revenir, vivre, jour, frère, mal, mort (provoqué probablement par l'expression «fête des morts »: on peut vérifier, en effet, que cette réponse a été donnée le 11 novembre 2013, au lendemain de cette fête, ceci expliquant cela). Ceci nous rappelle que même des associations originales (ici mort $\rightarrow$ fête) peuvent être recoupées avec d'autres qui vont dans le même sens (ici événement)

\section{Conclusion}

C'est de manière délibérée que nous n'avons pas évoqué ici les aspects théoriques des associations évoquées par les mots, car ils ont été largement développés auparavant [3436]. Au fil des ans, l'appellation en français de ce genre de dictionnaires a évolué, entre « dictionnaire associatif », «d'associations verbales », «d'associations lexicales ». Il semble que celui «d'associations évoquées par les mots », moins concis, reflète cependant mieux la nature de cet objet lexicographique. Nous concevons les dictionnaires d'associations comme des instruments de mesure, permettant d'affiner l'élaboration de réseaux lexicaux, mais nous préférons rester très prudents dans les interprétations des relations entre cette structure et la pensée, ou, a fortiori, la «mentalité » des locuteurs de telle ou telle langue. Par ailleurs, un des développements les plus intéressants de ces dictionnaires est l'étude comparative des associations chez les bilingues et les monolingues, car si tout bilingue reconnait être différent dans l'une et l'autre langue [37, 38], ces nuances sont très difficiles à évaluer.

\section{Références}

1. Ассоциативный словарь употребительной русской лексики / научный ред. А. В. Рудакова, И. А. Стернин. Воронеж, Истоки, 2011.

2.Гольдин, В. Е., Мартьянов, А. О., Сдобнова, А. П. Электронный русский ассоциативный словарь школьников, Компьютерная лингвистика и интеллектуальные технологии. 2009, Вып. 8 (15). С. 69-74.

3. Гуц Е. Н. Ассоциативный словарь подростка, Омск, 2004

4. Дебренн М. Французский ассоциативный словарь Т.1. От стимула к реакции. Новосибирск, 2010. 252 С.

5.Дебренн М. Французский ассоциативный словарь Т. 2. От реакции к стимулу, Новосибирск, 2010. 449 С.

6. Черкасова Г. А, Уфимцева Н. В. Русский региональный ассоциативный словарьтезаурус EBPAC. http://iling-ran.ru/main/publications/evras.

7. Леонтьев А. А. Словарь ассоциативных норм русского языка. М. 1977.

8.Пуиг С., Караулов Ю. Н., Черкасова Г. А. Ассоциативные нормы испанского и русского языков. М., Мадрид : Азбуковник, 2001.

9.Русский ассоциативный словарь. В 2 т. / Ю. Н.Караулов, Г. А. Черкасова, Н. В. Уфимцева, Ю. А. Сорокин, Е.Ф. Тарасов. Т. І. От стимула к рекции: Ок. 7000 стимулов. М.: АСТ-Астрель, 2002. 784 с. Т. ІІ. От стимула к рекции: Более 100000 реакций. М.: АСТ-Астрель, 2002. 992. 
10. Словарь обыденных толкований русских слов. Лексика природы. В 2 т. Т. 1. А - М (АБРИКОС - МУРАВЕЙ) (480 слов-стимулов) / Под ред. Н. Д. Голева, // Авторы составители Басалаева М. Ю., Воробьева М. Е., Голев Н. Д., Дударева Я. А., Замилова А. В., Ким Л. Г., Кишина Е. В., Кузнецова Т. Ю., Мельник Н. В. «Издательство Кемеровского университета», 2011.- 400 с

11. Уфимцева Н. В. и др. Ассоциативные нормы русского и немецкого языков. М.-Воронеж: Ияз РАН - ВГУ, 2004.

12. Уфимцева Н. В., Черкасова Г. А., Караулов Ю. Н, Тарасов Е.Ф. Славянский ассоциативный словарь: русский, белорусский, болгарский, украинский. M., 2004, 792 c.

13. Шапошникова И. В., Романенко А. А, Русский региональный ассоциативный словарь (Сибирь и Дальний Восток), т.1 От стимула к реакции, М. Московский институт лингвистики 2014

14. Шапошникова И. В., Романенко А. А, Русский региональный ассоциативный словарь (Сибирь и Дальний Восток), т. 2: От реакции к стимулу / Отв. ред. Н.В. Уфимцева М.: Московский институт лингвистики, 2015. 763с.

15. Pavlova A., Bezrodnyj M. How to Catch a Unicorn? The Image of the Russian Language from Lomonosov to Wierzbicka // Toronto Slavic Quarterly. №32. Toronto, 2010.

16. Sériot P. Oxymore ou malentendu? Le relativisme universaliste de la métalangue sémantique naturelle universelle d'Anna Wierzbicka // Cahiers Ferdinand de Saussure. №57. 2004. Р. 23-43 (Перевод на русский язык: Оксюморон или недопонимание? Универсалистский релятивизм универсального естественного семантического метаязыка Анны Вежбицкой // Политическая лингвистика 1(35). Екатеринбург, 2011. С. 30-40).

17. Sériot P. La pensée ethniciste en URSS et en Russie post-soviétique // Strates. №12. 2005. P. 111-125.

18. Sériot P. Le déterminisme linguistique en Russie actuelle // Patrick Sériot (dir.) La question du déterminisme en Russie actuelle [en ligne]. Lyon, ENS LSH, mis en ligne le 10 décembre 2008 (http://institut-est-ouest.ens-lsh.fr/spip.php?article156).

19. Дебренн М., Неогумбольтианство и ассоциативные словари // Вестник НГУ Сер. Лингвистика, межкультурная коммуникация. Том 10 вып 2, 2012 стр. 77-86

20. Руссо М. М, Неогумбольдтианская лингвистика и рамки «языковой картины мира» // Политическая лингвистика. - 2014. - № 1 (47). - С. 12 - 24.

21. Черкасова Г.А. Квантитативные исследования ассоциативных словарей // Общение. Языковое сознание. Межкультурная коммуникация. Сб. статей. / Под ред. Н.В. Уфимцевой, Т.Н.Ушаковой. М.- Калуга, 2005. с. 308-318.

22. Debrenne M. Variabilité diatopique des associations évoquées par les mots en Francophonie // CMLF-16 DOI 10.1051/shsconf/20162703002

23. Debrenne M. La conscience métalinguistique ordinaire et la linguistique naïve dans les travaux russes contemporains // Le discours et la langue, ${ }^{\circ} 6.12014$, p:19-30

24. Дебренн М. Лексические отношения в ассоциативных словарях французского языка // Вопросы психолингвистики 2016, вып. 1, №16 с.76-89

25. Lonsdale, Le Bras, A Frequency Dictionary of French, Core vocabulary for learners, London, New York 2009

26. De Deyne S., \& Storms G. Word Associations: Norms for 1,424 Dutch words in a continuous task. Behavior Research Methods, 2008, 40,198-205.

27. Горошко Е. И. Проблема проведения свободного ассоциативного эксперимента // Известия Волгоградского государственного педагогического университета, серия "филологические науки", №3. - 2005

28. Lafourcade M., Le Brun N., Joubert A. Collecting and Evaluating Lexical Polarity with a Game with a Purpose.// proc International Conference on Recent 
Advances in Natural Language Processing (RANLP 2015), Hissar, Bulgaria, September 5 $-11,2015,9 \mathrm{p}$

29. Ricard Solé, Bernat Corominas-Murtra, Jordi Fortuny La structure en réseaux du langage // Pour la science $\mathrm{N}^{\circ} 434$ decembre 2013

30. Караулов Ю. Н., Сорокин Ю. А., Тарасов Е. Ф., Уфимцева Н. В., Черкасова Г. А. Русский ассоциативный словарь. т. т.1-6, М.: Институт русского языка РАН, 1994-1998.

31. Avanzi M. Atlas du français de nos régions. Armand Colin, Paris. 2017, 160 p.

32. Морель, М.-А., Арсеньева, В. А. Синтаксис и застывшие формы. Морфосинтаксические закономерности свободных ассоциаций в эксперименте по созданию «Ассоциативного словаря французского языка» (пер. соавтора А. В. Арсеньевой) // «Вестник Новосибирского университета. Серия: Лингвистика и межкультурная коммуникация». - 2011. - Том 9 (вып.1). - С.83-92

33. Акованцева Н.В., Структура интегрального ассоциативного поля «отдых» в русском языковом сознании // Вестник ВГУ. Серия: Филология. Журналистика. 2015. No 4 стр. 5-6.

34. Debrenne M., Ufimceva N. L'apport des dictionnaires d'associations lexicales aux études de sémantique // Syntaxe et sémantique, $\mathrm{n}^{\circ}$ 12, 2011, p. 121-137

35. Debrenne M. Variabilité diatopique des associations évoquées par les mots en Francophonie // CMLF-16 DOI 10.1051/shsconf/20162703002

36. Debrenne M. La psycholinguistique en Russie et les dictionnaires d'associations évoquées par les mots // Revue française de linguistique appliquée 2017/1 Vol. XXII. P 7588

37. Dewaele J.-M. Why do so many bi- and multilinguals fell different when switching languages? // International Journal of multilingualism, 2015, pp. 1-14.

38. Dewaele J.-M., Nakano S. Multilingual's perceptions of feeling different when switching languages // Journal of Multilingual and Multicultural Development, 34(2), 107120. 